\title{
Roles of mitonuclear ecology and sex in conceptualizing evolutionary fitness
}

\author{
By Kyle B. Heine ${ }^{1} \&$ Elay Shech ${ }^{2}$
}

April 7, 2021

-Preprint. Forthcoming in Biology \& Philosophy

\begin{abstract}
We look to mitonuclear ecology and the phenomenon of Mother's Curse to argue that the sex of parents and offspring among populations of eukaryotic organisms, as well as the mitochondrial genome, ought to be taken into account in the conceptualization of evolutionary fitness.

Subsequently, we show how characterizations of fitness considered by philosophers that do not take sex and the mitochondrial genome into account may suffer. Last, we reflect on the debate regarding the fundamentality of trait versus organism fitness and gesture at the idea that the former lies at the conceptual basis of evolutionary theory.
\end{abstract}

1. Introduction. The concept of "fitness" is central to the foundations of evolutionary biology, especially as it ostensibly plays an explanatory role in how natural selection accounts for much of the diversity and adaptation of the biological realm (Orr, 2009; Rosenberg \& Bouchard, 2015). Famously, Herbert Spencer coined the phrase "survival of the fittest" in the $19^{\text {th }}$ century after reading Charles Darwin's On the Origin of Species and, upon the suggestion of Alfred Russel Wallace, Darwin adopted the phrase into the $5^{\text {th }}$ edition of his book in 1869 . Ronald Fisher (1958) conceptualized and developed much of the mathematics of fitness and

${ }^{1}$ Department of Biological Sciences, Auburn University, Auburn, AL 36849 USA. E-mail: kbh0039@auburn.edu

2 Department of Philosophy, Auburn University, Auburn, AL 36849 USA. E-mail: eshech@auburn.edu 
natural selection but did not appear to define fitness directly in his work (Grafen, 2020). ${ }^{3}$ The notion of fitness has led to much philosophical debate regarding its meaning, its quantification, to what entities it applies, what type of probabilities are involved, etc. (Millstein, 2017). Our goal in this paper is to contribute to such debate by exploring how evolutionary fitness can be conceptualized among populations of eukaryotic ${ }^{4}$ organisms that are the products of the coevolution of mitochondrial (mt) and nuclear (N) genomes, and thus focus on a lacuna in the literature that we think is important for understanding fitness. Namely (among populations of sexually-reproducing eukaryotic organisms), we submit that the sex of parents and offspring, as well as the mt genome, ought to be taken into account as a component of evolutionary fitness. Failure to do so can lead to misconceptions, and our suggestion here is to re-characterize fitness in a manner that takes sex into account. To simplify the explanations herein, we refer to fitness as a summary statistic as outlined by Orr (2009), as opposed to conceptualizing fitness as a value assigned to an individual token-organism (but more on this below).

Our basic idea can be outlined as two main steps. First, consider a simple, hypothetical scenario in which two organisms in a population, call these organisms "A" and "B", each have one trait, call these traits "A" and "B" respectively. Say that A has many offspring, and B has only one offspring. Intuitively, we would want to say that organism A is fitter than organism B, and that trait A confers greater fitness than trait B. So far so good. Now, consider further that trait $\mathrm{A}$ is a peculiar trait since, while organism $\mathrm{A}$ has many offspring in the $\mathrm{F} 1^{5}$ generation, it has

\footnotetext{
${ }^{3}$ As Grafen $(2020,9)$ notes, the possibility of alternative characterizations of fitness arises since, in Fisher's fundamental theorem of natural selection, "the term fitness, which occurs as 'mean fitness' on the left-hand side of the fundamental theorem, and as 'genetic variance of fitness' on the right-hand side, is not actually defined by Fisher anywhere in his book or, indeed, elsewhere." Furthermore, as discussed below, while Fisher already discussed important ideas in this paper, e.g., transgenerational aspects of fitness, we discuss fitness under current advancements in the field of mitonuclear ecology, which was not a field of study early in the $20^{\text {th }}$ century.

${ }^{4}$ Eukaryotes are organisms whose cells contain membrane-bound organelles, among other traits that differ from prokaryotic organisms.

${ }^{5}$ The F1 generation refers to the first filial generation.
} 
none in the F2 generation since trait A triggers infertility/sterility in F2 generation organisms. Organism B, on the other hand, has offspring in the F1 generation, F2 generation, and so on. We submit that, in such a scenario, it is clear that trait B confers greater fitness than trait $\mathrm{A}$. The question arises though whether such considerations actually matter for working biologists. After all, so the objection goes, all offspring of an organism may die out in some generation, and this in and of itself seems like a bad reason to hold that some trait is not "fit." What we show in this paper is that such considerations do matter for working biologists in thinking about fitness, namely, in the field of mitonuclear ecology and in the context of phenomena like Mother's Curse.

Specifically, and second, eukaryotic organisms contain both a mt genome that is typically ${ }^{6}$ inherited only from the mother and a $\mathrm{N}$ genome, one half of which is inherited from the father and one half from the mother. The survival, reproduction, and evolution of eukaryotic organisms relies on the co-function and coevolution of the mt and $\mathrm{N}$ genomes. The sex of both parents and offspring affects how $\mathrm{mt}$ and $\mathrm{N}$ genes, and gene products, will be founded and cofunction in subsequent generations, provided the $\mathrm{mt}$ genome is inherited only from the mother and does not recombine. Trait fitness is often conceptualized as how a trait influences both survival and reproduction with a given population (Arnold, 1983). Therefore, how a trait influences survival and reproduction within a population is directly influenced by how mt and $\mathrm{N}$ genes are transmitted and co-function differently between males and females. Such an approach to conceptualizing fitness is meant to play a causal-explanatory role instead of simply drawing the conclusion that "those that reproduce the most are most fit"; we need not limit our knowledge and concept of fitness solely to reproductive success. Lastly, understanding the roles of

\footnotetext{
${ }^{6}$ There have been few exceptions to this phenomenon. See Luo et al. (2018).
} 
mitonuclear ecology and sex in fitness can help solve the problem of what we can know regarding the reproductive success of offspring several generations in the future instead of focusing solely on the F1 generation.

The structure of the paper is as follows. In Section 2, we provide a brief overview of mitonuclear ecology and outline how traits may be directly, or indirectly, affected by coevolution and co-function of $\mathrm{mt}$ and $\mathrm{N}$ genes. We then apply this concept, in Section 3, to understand how the sex of parental and offspring generations influences trait fitness within a given population. Section 4 provides specific examples of how sex may directly influence trait fitness under a specific subfield of mitonuclear ecology. At this point, through the specific example of mitonuclear ecology, we hope to have shown the important role of sex and the mt genome in conceptualizing trait fitness in the context of evolutionary biology. Section 5 then shows how characterizations of fitness that do not take sex into account may suffer. Next, in hope to make a further connection with philosophical debates about fitness, Section 6 gestures at a potential application of our discussion to the question of whether organism or trait fitness is at the basis of evolutionary theory (Sober, 2013; Pence \& Ramsey, 2015). We don't pretend to settle the issue here. Instead, our aim is solely to show how taking sex and the mt genome into account may matter for some philosophical debates about fitness. In Section 7, we consider an objection to the effect that our account solely echoes the starting point of many accounts of fitness such as Michod (2000) and Ariew and Lewontin (2004). Section 8 ends the paper with a short conclusion.

Before starting in earnest, several caveats are in order. First, what do we mean by “traits?" Here, traits are referred to as any heritable phenotype that is influenced by one or more genes and environmental factors. The term trait does not necessarily refer to a particular 
genotype (although it can), but the manifestation of how one or more genotypes interact with each other and/or environmental factors (e.g., height, mitochondrial function). Second, there are at least two major ways that fitness has been conceptualized: organism and trait fitness. Individual token-organism fitness_-or, organism fitness for short—can be defined as the cumulative effect that all traits impose on an organism's ability to survive and reproduce. As stated previously, trait fitness focuses more on how an individual trait (or sometimes genotype) impacts survival and reproductive success. Herein, we focus on the concept of trait fitness, but we circle back to a comparison with organism fitness in Section 6. However (as we discuss further in Section 2), certain measures of trait fitness in practice, such as absolute fitness, can be equal to that of organism fitness, provided that each organism will express only one value for a given trait at a given time. Lastly, this study does not attempt to develop a mathematical explanation for how sex may be incorporated into calculations of fitness but instead focuses on how fitness should be conceptualized with respect to mitonuclear ecology and sex. We leave any mathematical developments to theoretical biologists and/or other philosophers of science. Nonetheless, any reasonable account of trait fitness - in order to also be useful to biologistsneeds to meet one of two conditions of adequacy: It must either describe the current state of some type (genotype, trait, etc.) within a population or predict evolutionary change. ${ }^{7}$ We will return to this point in Sections 4-5.

2. Overview of Mitonuclear Ecology. Ronald Fisher was largely successful at conceptualizing fitness early in the $20^{\text {th }}$ century. In particular, his genetical theory of natural selection played a

\footnotetext{
${ }^{7}$ Compare, for example, with Ariew and Ernst $(2009,290)$ two adequacy constraints: "(A) A fitness concept must be able to explain why one trait is expected to be better represented in a population under the influence of natural selection. ... (B) A fitness concept must enable us to compare the degree to which natural selection will favor the spread of one trait over another, alternative trait."
} 
substantial role in uniting Darwinian evolution via natural selection and the nature of inheritance (Fisher, 1958). Although his work considered sex as influencing fitness (viz., different classes of organisms can have different rates of increase in fitness), Fisher's theory was before the discovery of mt DNA and, therefore, before the discovery that mitochondria are inherited maternally. Consequently, how mitochondria are inherited differently between males and females, and how $\mathrm{mt}$ and $\mathrm{N}$ genes must co-function in eukaryotes, was not a direct consideration within his theory.

Generally, Fisher's principle explains why the sex ratio of most populations of sexually reproducing organisms is approximately 1:1 (Fisher, 1958, 141-145). ${ }^{8}$ Namely, organisms that produce $n$ offspring of the rarer sex will leave more grand-offspring and, hence, be more "fit" than organisms that leave $n$ offspring of the more common sex. More specifically, Fisher's idea of reproductive value addresses the problem of determining how an individual of a certain age and class (e.g., sex) will contribute to the production of future generations (Fisher, 1958, 27-30). The notion of reproductive value plays an important role in conceptualizing fitness by taking account of how offspring are distributed among classes, in addition to the number of offspring produced. To this end, although sex was clearly considered early in concepts of fitness, it was not considered with mitochondria and their unique form of inheritance in mind. The remainder of this paper proceeds under this acknowledgement and addresses the important roles of mitonuclear ecology and, therefore, sex in conceptualizing fitness. Moreover, the concept of reproductive value ${ }^{9}$ will be related to some of the points we discuss later in the study (e.g., multigenerational aspects of fitness), however, we update such discussions by framing fitness

\footnotetext{
${ }^{8}$ We thank an anonymous reviewer for noting the importance of Fisher's famous "grand-offspring" argument. However, see Edwards (1998) for sources that may have been used by Fisher in the development of this principle, such as Darwin's The Descent of Man and Selection in Relation to Sex (1896).

${ }^{9}$ For more information on reproductive value, see Grafen (2020) and references therein, including Lion (2018).
} 
within our understanding of mitonuclear ecology. Therefore, while we acknowledge that sex and a multigenerational view of fitness have been discussed previously to some extent (e.g., via Fisher's notion of reproductive value), we bring the reader's attention to how our current knowledge of mitochondria plays a critical role in conceptualizing fitness; for instance, how uniparental inheritance of mitochondria may change the way we think about the fitness of parents and grand-offspring.

A crucial constituent of evolutionary fitness, along with survival, is reproductive success - the ability of an organism to perpetuate its genes in future generations (Clutton-Brock, 1988). Including reproductive success as a component of trait fitness is reasonable since any consideration of fitness is incomplete provided a trait cannot make it into the next generation (i.e., survival is only important from an evolutionary perspective in so much as an organism must survive to reproduce). One factor that is often missing from this line of reasoning, however, is the sex of both parents and offspring. Given our current knowledge of mitonuclear ecology, whether an organism produces male or female progeny, and whether traits are influenced by genes that are mt or $\mathrm{N}$ encoded, ${ }^{10}$ has dire ramifications for how a trait will be founded and/or function in subsequent generations (Hill, 2019). If we are interested in the fitness of a given trait that is influenced by both $\mathrm{mt}$ and $\mathrm{N}$ genes (e.g., respiration), then the genes and gene products of both genomes must co-function ${ }^{11}$ well for the trait to be expressed successfully.

Generally, evolutionary fitness has been compared between populations (Lima et al., 2019) and between males and females within populations (Foerster et al., 2007). However, the

\footnotetext{
${ }^{10}$ The vast majority of research into evolutionary theory has focused on the $\mathrm{N}$ genome of eukaryotic organisms, but eukaryotes also contain an entirely separate genome in the mitochondrion. The gene products of the mt genome regularly interact with gene products of the $\mathrm{N}$ genome.

${ }^{11}$ Co-function of the $\mathrm{mt}$ and $\mathrm{N}$ genomes refers to the necessity of these two genomes to work together for oxidative phosphorylation (OXPHOS) to be carried out efficiently. This is due to the fact that some $\mathrm{N}$ genes that function in the mitochondrion must work alongside $\mathrm{mt}$ genes that also function in the mitochondrion; for example, genes that code for OXPHOS proteins of complexes in the electron transport system.
} 
effect that a trait has on survival and reproduction within a population will depend on how the trait is inherited and expressed-whether the trait is present, and how it functions, in female versus male individuals. Our understanding of how the $\mathrm{mt}$ and $\mathrm{N}$ genomes are inherited, interact, and co-function between females and males should be taken into account when conceptualizing evolutionary trait fitness. Hence, sex itself should be incorporated into the concept of trait fitness, as opposed to merely conceptualizing differences in fitness between females and males. Mitonuclear ecology is a field of evolutionary ecology that addresses the coadaptation and co-function of the $\mathrm{mt}$ and $\mathrm{N}$ genomes of eukaryotes (see Hill, 2019). The eukaryotic lineage began with a symbiotic union of two prokaryotes which each retained a genome in the new organism $^{12}$ (Lane, 2006). A new focus on the need for coadaptation of these two genomes has brought a mitonuclear focus to studies of adaptation, senescence, sexual selection, and speciation. What is becoming apparent in this new field of research is that the co-function of the products of these two genomes - in unison - is crucial to the survival and reproduction of eukaryotic organisms. The implication of this new mitonuclear emphasis in evolutionary ecology is that our current notion of trait fitness is incomplete.

Past concepts of fitness have correctly taken into account survival and reproductive success. Let us think about a toy example of natural selection in order to illustrate what various notions are at play. Consider an inverse pyramid of slots that get increasingly smaller towards the bottom (similar to the game Connect Four, but an inverted pyramid instead of a square). Genotypes within the population are represented by coins of varying size that can pass through the slots. Larger coins (lower fitness) will remain at the top of the inverse pyramid, and smaller

\footnotetext{
${ }^{12}$ The eukaryotic cell was likely formed when an archaeon engulfed a bacterium approximately two billion years ago, where the archaeon genome became the $\mathrm{N}$ genome, and the bacterial genome became the $\mathrm{mt}$ genome. These genomes have been largely modified over the course of evolutionary history with the archaeon genome increasing to $\sim 20,000 \mathrm{~N}$ genes, and the bacterial genome being reduced to $37 \mathrm{mt}$ genes.
} 
coins (higher fitness) will pass closer to the bottom of the pyramid. Let coin thickness represent survival and coin diameter represent reproductive success. Therefore, in order for each coin to pass through a given slot and move closer to the bottom, it must meet the minimum thickness (ability to survive) and diameter (ability to reproduce) required. Dimes, pennies, and quarters represent three genotypes. $25 / 100(25 \%)$ of the population are dimes, $50 / 100(50 \%)$ are pennies, and $25 / 100(25 \%)$ of the population are quarters. If you are a dime, let your chance of reproducing be 0.8 since you have a small diameter and are thin. If you are a penny, let your chance of reproducing be 0.5 since you are of a medium diameter and medium thickness. If you are a quarter, let your chance of reproducing be 0.2 because you are of a large diameter and large thickness. Depending on the manner in which fitness is viewed, we calculate different values of fitness either among organisms or genotypes within a given population (see Table 1).

Two key components of mitonuclear ecology are missing from these current models of fitness: the sex of parents and offspring, and the mt genome. In some respects, these two components go hand-in-hand, provided that the mt genome is typically only inherited from one's

Table 1 | An outline of how different fitness types have been conceptualized and applied in evolutionary biology (Orr, 2009) and whether or not those types correspond to concepts of organism (Pence \& Ramsey, 2015) or trait (Sober, 2013) fitness in philosophy of biology. We provide a toy example for each fitness type.

\begin{tabular}{|c|c|c|c|}
\hline Fitness type & Organism & Trait & Example \\
\hline $\begin{array}{l}\text { Absolute: a genotype's total fitness-the } \\
\text { collective effects of a genotype on survival } \\
\text { and reproductive success }\end{array}$ & No & Yes & $\begin{array}{l}\text { Dime absolute fitness: } 0.80 \\
\text { Penny absolute fitness: } 0.50 \\
\text { Quarter absolute fitness: } 0.20\end{array}$ \\
\hline $\begin{array}{l}\text { Relative: absolute fitness normalized to the } \\
\text { absolute fitness of the fittest genotype }\end{array}$ & No & Yes & $\begin{array}{l}\text { Dime relative fitness: } 0.80 / 0.80=1.00 \\
\text { Penny relative fitness: } 0.50 / 0.80=0.625 \\
\text { Quarter relative fitness: } 0.20 / 0.80=0.25\end{array}$ \\
\hline $\begin{array}{l}\text { Individual: the survival and reproductive } \\
\text { success of an organism }\end{array}$ & Yes & No & $\begin{array}{l}\text { Individual with dime: } 0.80 \\
\text { Individual with penny: } 0.50 \\
\text { Individual with quarter: } 0.20\end{array}$ \\
\hline $\begin{array}{l}\text { Mean: the sum of fitnesses (absolute, } \\
\text { relative, or individual) within a population } \\
\text { under Hardy-Weinberg equilibrium: } \\
\bar{W}=\mathbf{p}^{2} \boldsymbol{w}_{\mathbf{1}}+\mathbf{2} \mathbf{p q} \boldsymbol{w}_{\mathbf{2}}+\mathbf{q}^{\mathbf{2}} \boldsymbol{w}_{\mathbf{3}} \text {, where } \mathbf{p}^{\mathbf{2}} \text { is the } \\
\text { frequency of dimes, } \mathbf{2} \mathbf{p q} \text { is the frequency of } \\
\text { pennies, } \mathbf{q}^{2} \text { is the frequency of quarters, and }\end{array}$ & Yes & Yes & $\begin{array}{l}\text { Absolute or individual mean fitness }= \\
0.25(0.80)+0.50(0.50)+0.25(0.20)= \\
0.20+0.25+0.05=0.50 \\
\text { Relative mean fitness }= \\
0.25(1)+0.50(0.625)+0.25(0.25)= \\
0.25+0.3125+0.0625=0.625\end{array}$ \\
\hline
\end{tabular}


$\boldsymbol{w}_{\mathrm{i}}$ are the fitnesses of the genotypes or

individuals

mother, and therefore, is only passed down through female progeny. This raises two concerns with conceptualizing fitness. First, fitness is not only influenced by the number of offspring in the F1 and subsequent generations, but by the sex of those offspring. Furthermore, it may be that we cannot know how well an organism's offspring will be at surviving and reproducing many generations later. Many biologists, in practice, calculate fitness from zygote to zygote (i.e., as the ability of an organism to produce viable offspring), however, this practice raises concern, provided that an organism's offspring are not guaranteed to have the same reproductive success as either parent. Taking sex into account helps solve this problem (see Section 3 below). Second, whether or not traits of interest are influenced by the mt genome will have an impact on the propensity ${ }^{13}$ of natural selection to influence a population, provided the mt genome does not undergo the same mixing of alleles as the $\mathrm{N}$ genome. Since the mt genome of bilaterian animals typically does not recombine, the genetic hitchhiking ${ }^{14}$ of deleterious alleles can lead to selective sweeps within a population (Hill, 2020) and influence trait fitness. Natural selection acts on the entire mt genome as a single unit and not individual genes, possibly leading to mutational erosion and compensatory coevolution within a population.

Mother's Curse (Gemmell et al., 2004) is one example of how differential fitness between males and females can develop when deleterious (harmful) alleles accumulate in the mt genome. Since the mt genome is not inherited paternally, males are a dead end for mt genes, so

\footnotetext{
${ }^{13}$ Here, propensity refers to a causal notion of probability, i.e., a "difference maker" or an aspect of a population that invokes change, as outline by Sober (2013).

${ }^{14}$ Genetic hitchhiking occurs when deleterious alleles are inherited along with beneficial alleles since the mt genome does not recombine. As a result, the frequency of the deleterious alleles changes within a population based on selection of the beneficial alleles with which it is associated. If strong selection on one allele leads to the fixation of that allele plus all of the alleles with which it is associated, it is known as a selective sweep.
} 
their mt genomes are not subject to natural selection. Alleles that are detrimental to males, and neutral or beneficial to females, become fixed in maternally inherited mt DNA (Vaught \& Dowling, 2018). As a result, the fitness of a given trait may be different when sex is taken into account, as opposed to looking at fitness across a population as a whole (Dowling \& Adrian, 2019; Nagarajan-Radha et al., 2020). Therefore, the sex of both parents and offspring plays a crucial role in determining the fitness of a given trait that is expressed in both sexes but functions differently in males than females.

3. Parental and Offspring Sex. From an evolutionary perspective, both the short and long-term goal $^{15}$ of an organism is to pass its genes to subsequent generations. Many accounts of fitness to date have taken into account the ability of an individual organism to survive to reproductive age, along with the total number of offspring that an organism produces in its lifetime; for example, when Brandon (1978) and Mills and Beatty (1979) discuss fitness for individual organisms in terms of the expected number of offspring. However, one component of fitness that has gone largely unaddressed is the ability of genes to reach beyond the F1 generation. ${ }^{16}$ In other words, an organism that produces one fertile offspring — which itself can and does go on to reproduceshould have greater fitness over an organism that has five offspring, of which none reproduce. ${ }^{17}$ This difficulty in calculating fitness estimates for traits is further complicated by the sex of those offspring and whether said traits are mt encoded, $\mathrm{N}$ encoded, or some combination of the two.

\footnotetext{
15 Of course, organisms do not truly have "goals" in the sense that they plan how many offspring they will produce and how long they will live. Here, the term "goal" refers to the fact that it is necessary for organisms to survive to reproductive age and reproduce in order to pass their genes to subsequent generations.

${ }^{16}$ But see Ramsey (2006) and Pence and Ramsey (2013) for notable counterexamples.

${ }^{17}$ Quantitative genetics has certainly incorporated pedigrees and life history traits in models of selection (see Hadfield \& Nakagawa, 2010), but here, we focus specifically on considerations of the mt genome and sex in conceptualizing fitness.
} 
For instance, let us take respiratory function as a trait of interest. Those individuals within a given population that have a greater respiratory efficiency are typically considered to be "more fit", provided that those individuals may be better at supporting their own growth, selfmaintenance, and reproduction (Harada et al., 2019; Heine \& Hood, 2020). Based on all the individuals within a given population, we may want to determine the optimum respiratory function provided a certain combination of $\mathrm{mt}$ and $\mathrm{N}$ genes. Respiratory function is controlled by the electron transport system (ETS) within mitochondria of both male and female individuals. A certain number of $\mathrm{mt}$ and $\mathrm{N}$ genes that code for ETS complexes within mitochondria must work together for mitochondrial respiration to work efficiently. Within mitochondria, energy is produced by creating an electrochemical gradient across the inner mitochondrial membrane. Protons are actively pumped from the mitochondrial matrix into the inter-membrane space by complexes I, III, and IV, as complexes I and II deliver electrons to the quinone pool, and complex IV reduces oxygen to water. Protons then flow down their electrochemical gradient and through complex V to convert adenosine diphosphate to adenosine triphosphate (Hatefi, 1985). This is where the sex of offspring comes in to play.

If a deleterious, mt mutation were to arise through replication error in the germ line of a female individual, whether or not that error propagates throughout the population will be influenced by the sex of her offspring (and grand-offspring), and consequently, change the fitness estimate of that trait within the population. However, this fitness estimate cannot be known until the female reproduces.

As previously stated, in bilaterian animals, mitochondria are typically inherited strictly from one's mother. If we take two possible instances of reproduction for our hypothetical female, we can see how this may potentially unfold: 
Scenario $1\left(\mathrm{~S}_{1}\right)$ : Our hypothetical female produces one female offspring that goes on to produce two male and two female offspring of her own.

Scenario $2\left(\mathrm{~S}_{2}\right)$ : Our hypothetical female produces one male offspring that goes on to produce one male and one female offspring of his own.

In $S_{1}$, the deleterious mutation will not only make it into the $\mathrm{F} 1$ generation, but will be passed down to both the two granddaughters and two grandsons in the F2 generation. In this scenario, although more grand-offspring are produced, we would say that the optimum fitness estimate of respiratory function is less than ideal, or reduced, given that the mutation will be founded in subsequent generations and will have a negative influence on individuals beyond the F1 generation. In $\mathrm{S}_{2}$, however, the deleterious mutation will again make it into the $\mathrm{F} 1$ generation but not into the F2 generation. This is due to the fact that the male son in the F1 generation will not pass the mutation down to his offspring (the grand-offspring) in the F2 generation, provided that the mutation is in the mt genome. ${ }^{18}$

These hypothetical scenarios demonstrate how the fitness of a trait within a given population is not only dependent on the number of offspring produced, but is dependent on the sex of both parents and offspring. Accordingly, not only is it necessary to incorporate sex into concepts of trait fitness, but we must also consider how a trait is encoded and by what genes it is affected. The next section explains how sex-specific trait mutations that are encoded in the $\mathrm{mt}$ genome, in particular, are problematic for conceptualizing evolutionary trait fitness.

\footnotetext{
${ }^{18}$ This will work differently if the mutation is on an $\mathrm{N}-\mathrm{mt}$ gene (a $\mathrm{N}$ gene that functions in the mitochondrion), which can be passed down to offspring from both male and female parents. This will also be largely influenced by $\mathrm{XY}$ versus ZW mating systems.
} 
4. Mother's Curse. "Mother's curse" is a form of dysfunction that arises where male-specific mt DNA mutations are neutral or beneficial to females and, therefore, selection does not act on such mutations since males do not transmit their mt DNA to their offspring. In any species in which there is exclusively female transmission of $\mathrm{mt}$ genes, male-specific mutations that arise in the $\mathrm{mt}$ genome are difficult to eliminate through natural selection. Deleterious, mt mutations can accumulate within a mother's mt genome and are passed down from one generation to the next, unchecked by selective pressures. Such effects should be considered if variation in trait fitness is to meet one of the two conditions of adequacy that we have stated above and be useful to working biologists, namely, successfully predicting evolutionary change in subsequent generations, or summarizing the current state of fitness among individuals within a given population.

If a deleterious, mt mutation that affects male sperm motility arises in a female parent, the difficulty in conceptualizing fitness reaches beyond the sex of offspring and how the gene is inherited. The issue now becomes how the trait affects males and females separately. The mutation can remain present in the population where it may continue to decrease the reproductive success of males but not females. With respect to trait fitness, difficulty arises because the trait can now readily be passed down the female line with little selective pressure since it does not directly affect the female herself. Hence, if we are to determine the optimum reproductive success within a population provided sperm motility and function, variation in trait fitness must take into account that the trait may directly influence male but not female progeny.

Producing more female offspring may increase an individual's lifetime reproductive success and, therefore, the overall trait fitness within a population even at the expense of males. 
However, such a scenario becomes further complicated by the fact that deleterious mutations to the mt genome that hinder male reproductive performance may also reduce male longevity (Camus et al., 2012). Although it may seem as though such an effect can proliferate throughout a population indefinitely, $\mathrm{N}$ restorer genes ${ }^{19}$ that reverse such negative effects in males can evolve. This possibility further corroborates the need to understand how both the $\mathrm{N}$ and $\mathrm{mt}$ genomes act together to influence males and females separately within a population.

5. Reconceptualizing Trait Fitness. In this section, we wish to illustrate how our discussion up until now, vis-à-vis the pertinence of sex and the mt genome to the concept of fitness, applies to recent discussions of fitness in the philosophical literature. For example, Pence and Ramsey (2015, 1083-1085) present the following three concepts of trait fitness:

(TF1) The fitness of a trait $t$ is equal to the average individual (organismic) fitness values of individuals bearing $t$.

(TF2) The fitness value of a trait is a quantity that is, given some model of population dynamics, predictive of the future dynamics of that trait in a population.

(TF3) Trait fitness is the reproductive advantage to the individual conferred by possessing the trait.

By recalling our previous hypothetical scenario from Section 3, we can see how not taking sex into account leads to inaccurate and misleading accounts of trait fitness. Since these three accounts of trait fitness are closely related to one another, it is most clear to begin with

\footnotetext{
${ }^{19} \mathrm{~N}$ restorer genes are nuclear genes that evolve to counteract the detrimental effects of the mt genome. Such restorer genes seem to be rather widespread among eukaryotes.
} 
TF3. With respect to TF3 and our hypothetical scenario, TF3 would predict that all individuals such as the female in $\mathrm{S}_{1}$ would confer a greater fitness advantage over individuals such as the male in $\mathrm{S}_{2}$ by having four offspring in comparison to the male's two offspring (i.e., the female would have a "reproductive advantage"). However, we know from our example that all four offspring from the female will carry a deleterious, mt mutation. Therefore, although the male only produced two offspring, values of trait fitness calculated from the phenotypes of his offspring should hold a greater value since they would not carry the deleterious mutation as the four offspring from the female. This point can only be addressed if sex (and mt genotype) of the parental generation is taken into account instead of solely accounting for reproductive advantage (i.e., number of offspring), as is the case for the current concept of TF3.

One objection to this point is to say that if the female in $S_{1}$ carries a deleterious, $\mathrm{mt}$ mutation, then that should be reflected in her inability to produce ample offspring, and sex need not be important. Our response is that reproductive success is influenced by variation in both genetic and environmental factors. Therefore, the only way to truly determine the appropriate value of trait fitness would be to understand how the mutation will be founded in subsequent generations while also taking into account the respiratory function (in this case) of offspring. Admittedly, this does not give us an end-all, definitive answer to predicting evolutionary change, however, it does bring us one step closer to providing an explanatory role for the concept of fitness. The importance of sex in TF1 and TF2 is more subtle.

The incorporation of sex and mitonuclear ecology into TF1 and TF2 leads to an explanatory and more accurate understanding of the role of fitness. Here, it is true that the fitness of a trait is equal to the average organismic fitnesses of individuals bearing the trait; however, unless sex is taken into account, the fitness values will not be explanatory and predictive of 
future population dynamics, as stated in TF2. ${ }^{20}$ In our hypothetical scenario, without understanding that the mt mutation will affect the grand-offspring differently, born from the female in $S_{1}$ and male in $S_{2}$, any prediction of future population dynamics would be inaccurate. In relating respiratory function to reproductive success in our scenario, researchers may obtain a value for trait fitness, however, this will not provide us with any explanatory information for why it is the case that a certain respiration value leads to a certain reproductive value unless we understand that sex plays a causal role. Taking sex into account can tell us why respiration impacts reproductive success in a certain manner. Again, because not all of the grand-offspring would carry the deleterious mutation, the fitness values of the male and female parents should not solely differ based on respiratory function and number of offspring. Since we understand how the mutation will be passed beyond their offspring's generation, the parent's corresponding values of trait fitness (respiratory function, as it relates to reproductive success) should take into account that all of the female's offspring will carry the mutation, but the male parent will not pass the mutation to his offspring (unless he happens to mate with a female that does carry the mutation). Therefore, in order for the trait fitness value to be an accurate average (including males and females) of organismic fitness values (TF1), it must account for the fact that the trait will be expressed differently in future population dynamics (TF2) between males and females. ${ }^{21}$

As we stated in Section 1 and above, any reasonable account of fitness must meet one of two conditions for the notion to be considered an adequate account of trait fitness. It must either

\footnotetext{
${ }^{20}$ As should be clear from our elaboration on this issue, we are not appealing to some special account of explanation, although causal, counterfactual, and/or probabilistic accounts of explanation could easily fit the bill. Our point is that on a basic and intuitive level of what is meant by explanation, taking sex and the mt genome into account is indispensable for a faithful representation of fitness in this scenario and an accurate understanding of the evolutionary forces at play in the discussed situation.

${ }^{21}$ It is worthwhile to note that Pence and Ramsey (2015) do stress that TF1-TF3 are not equivalent and hint at some of the ideas that we develop here. For example, they note (1085): "If a trait has a significant benefit to individual organisms, yet is not (or not efficiently) transmitted from parents to offspring, then the TF3-fitness of that trait may be high while its TF2-fitness remains low."
} 
be predictive of evolutionary change or summarize the current state of a fitness type within a population. For either of these two conditions to be met, we must recharacterize our concept of trait fitness, and to do so must account for the fact that evolving populations of sexuallyreproducing eukaryotes contain different fitness types for both males and females. Supporters of trait fitness often quantify trait fitness as the average of fitnesses among all organisms within a population at a given time (Mills \& Beatty, 1979; Bouchard \& Rosenberg, 2004; Sober, 2013). However, as we noted in our preceding discussion, this notion of trait fitness is incomplete—and inaccurate - in populations of sexually-reproducing animals provided that the fitness types of males and females are inherently different and predictive of future population dynamics. Admittedly, the concepts herein are focused on sexually-reproducing eukaryotes and will not be applicable to some other types of organisms (e.g., bacteria).

In addition, we wish to attend to a possible concern. Why should practicing biologists' work concern itself with the mt genome when most measures of survival and reproductive success will have nothing to do with mt genes? The answer to this worry is that very few aspects of animal survival and reproductive success could be carried out successfully without efficient mitochondrial function (i.e., animals need to produce energy efficiently to survive and reproduce at all). For further explanations of how mitochondria are ubiquitously linked to whole-animal performance, we refer the reader to Heine and Hood (2020).

The most crucial aspect of our thesis is the subtle point that while TF1-TF3 may provide practicing biologists with correct values of trait fitness, not taking sex into account will (1) lead to values of trait fitness that do not play causal roles in understanding natural selection (i.e., calculating the fitness value of a trait will not provide researchers with a mechanism that explains selective pressures) and (2) lead to values of trait fitness that are not accurately 
predictive of future population dynamics. Incorporating sex as a component of evolutionary trait fitness accomplishes both of these goals which we claim are the ultimate objectives of conceptualizing trait fitness. With respect to the first point, we can calculate an optimum value for a trait that leads to increased reproductive success, but unless we understand how that trait is influence by the strict maternal inheritance of mt genes, we may not fully comprehend the causal aspect of why the frequency of that trait in particular changes across generations with certain sex ratios. It is one thing to demonstrate that the fitness of a trait differs between males and females across generations; it is another notion altogether to explain why this is the case. Such a model of trait fitness draws a line between descriptive and causal roles of fitness. The two aims are also of utmost importance to practicing biologists.

Last, it is worthwhile to end by making another connection between our discussion and the philosophical literature. Specifically, Abrams $(2012,1)$ distinguishes between four different notions of fitness: “...statistical type fitness, which can be measured from population data, and parametric type fitness, which is an underlying property estimated by statistical type finesses," where these are two kinds of trait fitnesses. Next, “...measurable token fitness, which can be measured on an individual, and tendential token fitness, which is assumed to be an underlying property of the individual in its environmental circumstances," are two kinds of organism fitnesses. We believe that the criticism leveled above against TF1-TF3 can be extended to cover statistical type fitness and measurable token fitness, as well as some instances of tendential token fitness. For example, Abrams (2012, 7) examines Bochard and Rosenberg's (2004) definition of fitness (as a type of tendential token fitness) in terms of the degree to which design problems are solved by a particular organism's traits. It is not clear that such design problems, insofar as they are solved by a particular organism's traits, allow for the kind of considerations of sex and the mt 
genome that we describe above (and so Bochard and Rosenberg's (2004) characterization of fitness may be lacking from our perspective). Interestingly though, the notion of parametric type fitness, which Abrams argues is the only notion that can play a causal and explanatory role in evolution, does seem to implicitly incorporate the type of issues that we discuss here. In particular, here is how Abrams (2012, 8-9) describes parametric type fitness:

We might view a population and its environment at a given time as represented by a point in a high-dimensional state space. This state space would represent the genotypes, phenotypes, locations, and internal physiological states of members of the population and of other organisms in the environment. It could also include the positions and states of abiotic elements that might affect survival and reproduction of members of the population. The state space would thus include all combinations of conditions which are relevant to the evolution of the population over the period of time under study ... We can then think of changes in the population and environment as a trajectory through this state space.... For competing heritable types, the population at any one time will include a distribution of organisms with those types. Parametric fitnesses then summarize probabilities of possible trajectories through the state space: for example, trajectories in which higher-fitness types increase in frequency would be more probable.

If we understand Abrams' idea correctly, he has set things up in a way that parametric fitnesses already take into account anything that may reasonably be relevant to the notion of evolutionary fitness. This would naturally include sex and the mt genome, and the type of multigeneration viewpoint that we emphasize above will be represented by the trajectories 
through this state space. In other words, by thinking about our scenarios $\mathrm{S}_{1}$ and $\mathrm{S}_{2}$ (from Section 3), the notion of parametric fitness will allocate higher probabilities to trajectories like $S_{2}$ instead of $S_{1}$, and so such a notion will not be amenable to the criticism that we present here. Instead, our contribution can be taken as identifying additional essential elements of the higher-dimensional space on which parametric type fitnesses are defined.

6. Organismic Versus Trait Fitness. The goal of this section is to gesture at how conceptualizing fitness in accordance with sex and the mt genome may have implications for philosophical debates about fitness. One such debate concerns whether the notion of organismic fitness or trait fitness lies at the foundation of evolutionary theory, with Sober (2013) siding with trait fitness and Pence and Ramsey (2015) with organismic fitness. To be clear, it is not our aim here to settle the debate. We simply wish to show that considerations discussed in this paper may be applicable since we suggest they shift the balance, so to speak, to the side of trait fitness as the concept that is more fundamental.

For brevity's sake let us concentrate on the "conceptual role of fitness," noted by Pence and Ramsey $(2015,1082)$, where the idea is that "fitness plays some sort of causal or explanatory role in the theory of natural selection." In this context, Pence and Ramsey $(2015,1082-1083)$ argue that various notions of trait fitness, such as TF1-TF3, are conceptually parasitic on the notion of (individual-token) organism fitness, "making [organism] fitness the fundamental notion of fitness in the conceptual role" such that "organismic fitness lies at the conceptual basis of the theory of evolution by natural selection." Starting with TF3, trait fitness is characterized by the manner for which it confers a reproductive advantage to the individual organism, and this in turn necessitates "the construction of a model of individual fitness" (1087). Similarly, TF1, stating simply that trait fitness is the average of organism fitness, and TF2 are derivable from organism 
fitness but not vice versa. Moreover, considering TF2, Pence and Ramsey $(2015,1087)$ maintain that "when TF2 is analyzed, individual fitness is one of its core components, but not vice versa."

However, in our view, as illustrated above in Section 5, when TF1-TF3 are fleshed out in terms of the organism fitness of actual individuals, while ignoring the role that sex and the mt genome play, the fitnesses calculated lead to results that are prima facia wrong or, at least, highly counterintuitive. After all, if organism fitness lies at the conceptual basis of evolutionary fitness, why would sex and the mt genome matter? On the other hand, if evolutionary fitness is constituted by how traits promote survival and reproduction over several generations, then it is clear that sex and the mt genome matter. Since sex and the mt genome matter to practicing biologist when dealing with fitness, as we have argued, this (inductively) supports the idea that trait fitness is more fundamental than organism fitness.

Furthermore, Pence and Ramsey $(2015,1088)$ note that while "it is true that organisms are built out of traits, and it is these traits that crucially determine organismic fitness, it is not true that trait fitness determines organismic fitness values." Although it is not the case that individual trait fitness values determine individual organismic fitness values, it is the case that variation in trait fitness determines organismic fitness. For instance, in Table 1, if we consider an extreme counterfactual scenario whereby the mean absolute fitness of a mt genotype is zero within a given population (i.e., a mt genotype causes male sterility across an entire population), then we should be able to imagine a scenario where we could still calculate a non-zero value of mean individual fitness for males within the same population. However, this is not the case, as these values should be equal to one another if they are based on reproductive success. If we imagine the same scenario, but the trait does not cause male sterility, then we would measure non-zero values of organismic fitness for males within the population. We do not claim that trait fitness 
alone determines organismic fitness, since organismic fitness is a culmination of the effects of many traits, but variation in trait fitness is essential to organismic fitness. ${ }^{22}$ Our main point here then is that such variation in trait fitness, and therefore organismic fitness, can only be estimated accurately by taking sex and the mt genome into account.

Nonetheless, if one rejects this line of thought, a proponent of the fundamentality of organism fitness owes us an explanation for why sex and the mt genome matter in the conceptualization of fitness if organism fitness lies at the conceptual basis of evolutionary theory. In either case then, we submit that a discussion of the role of sex and the mt genome is needed. ${ }^{23}$

7. Objections and Replies. The literature on contemporary fitness is large, while we have interacted with only a small part in this paper. As an anonymous reviewer notes, the basic issue concerning fitness is that Darwin's idea that the direction of evolutionary change depends solely on the natural properties of organisms and their environment is incomplete. There are extra considerations required to predict and understand evolution that are consequences of the mechanisms of inheritance and the details of reproductive schedules, among many other things, e.g., size of the population, mutation rates, migration rates, whether generations are overlapping or discrete. This is the starting point for many accounts of fitness such as Michod (2000) and Ariew and Lewontin (2004), so that one may object that we have done no more than echo this

\footnotetext{
${ }^{22}$ See Sober (2013) on the importance of variation in trait fitness.

${ }^{23}$ Perhaps it is worthwhile to add that, if our assessment in Section 5 is correct, and Abrams' (2012) notion of parametric type fitness is one that takes essential components like sex and the mt genome into account, and if Abrams' (2012) own argument to the effect that only parametric type fitnesses cause and explain evolution is correct, then there is another clear sense for which trait fitness is more fundamental than organism fitness (on the conceptual level). Namely, it is trait fitness that causes and explains evolution, not organism fitness.
} 
starting point, thereby overall obscuring whether there is an original contribution on our part. We have four replies to this line of thought.

First, while it is true that there are various mechanisms of evolution, such as mutation, genetic drift, and gene flow that extend beyond Darwin's idea of natural selection, our point about incorporating sex and the mt genome into accounts of fitness lies squarely within the realm of natural selection and mutation. We are thus not echoing the idea that these mechanisms are important; instead, we are illuminating causal aspects of fitness that are crucial to understanding natural selection and mutation. Second, our thesis does not merely echo the starting point for conceptualizing fitness, provided that sex and the mt genome are part of a larger, causalexplanatory story for understanding the role of fitness in evolutionary theory, i.e., such information can be used to understand how evolution occurs, as opposed to simply stating that some organisms or types are "more fit" than others. Third, related to contributions on our part, we have already noted the consequences of our paper for debates on fitness. Specifically, we have shown that characterizations of fitness that do not take sex and the mt genome into account may suffer, concentrating on Pence and Ramsey (2015) and also gesturing at accounts of Bochard and Rosenberg (2004) and Abrams (2012), and we have commented on how we view our account as generally supporting Sober's (2013) position of taking trait fitness as fundamental.

Last, in an effort to further connect with the literature and assuage worries as noted above, we will discuss how our work relates to some additional contributions in the literature. Again, we do not pretend to settle such debates here — such work must await a further occasionand it is of course beyond the scope of this paper to interact in detail with the admittedly sizable literature on the topic. 
There is a well-known debate in the literature between "causalists" (e.g., Millstein, 2006), who view forces of evolution like natural selection and drift as higher-level causes of evolutionary change, and "statisticalists" (e.g., Matthen and Ariew, 2002), who deny such a causal interpretation and ground evolution in events and interactions of individual organisms. Statisticalists, for instance, often argue that whether natural selection or drift take place depends on arbitrary choices, but causal factors are not arbitrary. Strevens (2016) in particular discusses such issues in relation to the reference class problem corresponding to the distinction between natural selection and drift. Specifically, one can distinguish between parameters and variables when discerning selection from drift: parameters are physical quantities that are taken into account when predicting an outcome and determining probabilities, and variables can be defined as those quantities that are not taken into account and thus have no influence on the probability of an outcome. Such line of thought is relevant in determining whether sex and the mt genome are causal, relevant aspects of conceptualizing fitness. Mitonuclear ecology teaches us that the sex of parents and offspring play causal roles in how mt genes are inherited in subsequent generations and, therefore, how working biologists can determine and predict probabilities. They are "difference-makers" and thus explanatory on Strevens' account. Hence, we can think of sex as a parameter (not a variable), provided that it plays a direct, causal role in the outcome of how genotypes are present in a given generation. Strevens $(2016,165)$ states that “...it is not things but the properties of things that are difference-makers..." In this regard, we must understand the properties of offspring to truly understand evolutionary change and conceptualize fitness, and our paper suggests one such avenue of study. Of course, this is not to say one cannot maintain a statisticalist account of evolution. Indeed, our study is partially sympathetic to aspects of the statisticalist position such as taking trait fitness as primitive. Rather, the point is that there may 
be further work needed to accommodate our study regarding which position one takes in the debate.

Similarly, our thesis can help solve (in part) some of the confusions of fitness present with fertility models. Ariew and Lewontin (2004), for instance, state that if fertility and viability are considered, assigning fitness values to genotypes is exceedingly difficult provided that offspring genotypes depend not only on the genotypes of a given individual but also on the genotype of its mate. This, however, is not the case for the $\mathrm{mt}$ genome of sexually-reproducing eukaryotes that exhibit strict maternal inheritance of $\mathrm{mt}$ genes. Here, we can predict (to an extent) the inheritance of $\mathrm{mt}$ genes and relative frequency of a type beyond the F1 generation, provided we have information on the sex of offspring. In this sense, sex offers a propensity account of fitness (Sober, 2013), in that whether or not a given trait is encoded (or influenced) by $\mathrm{mt}$ genes in a male or female will directly influence the presence and/or expression of that trait in offspring. The sex of parents and offspring can, therefore, directly impact survival and reproduction when considering phenomena like Mother's Curse.

Michod (2000) states that one of the things that we need to understand in order to comprehend fitness is how different degrees of fitness emerge throughout evolution. As such, it is not until the sex of parents and offspring (or generally, mitonuclear ecology) is taken into account that we can understand how evolution is operating and assign a causal role to evolutionary fitness. In addition, in reference to Fisher's rate of increase of a type, Michod $(2000,10)$ states that in addition "to depending on the environment and the composition of the population, a genotype's rate of increase in a sexual population depends upon the properties of the genotypes of all potential mates.” The fact that $\mathrm{mt}$ genes are inherited maternally within populations of eukaryotes is a property of mt genotypes that is relevant to understanding the rate 
of increase in said genotypes. Finally, Michod $(2000,187)$ states that, in certain cases, “...the concept of fitness must be extended into the realm of transmission and heritability. The challenge is how to do it." Our thesis lends a new solution to this problem. By taking mitonuclear ecology and sex into account when conceptualizing fitness, fitness can assume a causal role in select aspects of evolutionary theory.

8. Conclusion. Fitness is a fundamental notion in the context of evolutionary theory, and one that is taken to play an important causal and/or explanatory role. It is also important to actual working biologists in the research that they undertake. In this paper, we have argued for the significance of sex and the mt genome in the conceptualizations of evolutionary fitness by looking to the field of mitonuclear ecology and phenomena like Mother's Curse. We also gestured at the connection with the philosophical literature on fitness, and our hope is that our discussion can perhaps facilitate further advancement in the understanding of evolutionary fitness among philosophers. Future work by theoretical biologists and philosophers alike may find it useful to develop the mathematical and conceptual framework by which our thesis can be incorporated into calculations of fitness.

\section{REFERENCES}

Abrams, M. 2012. Measured, modeled, and causal conceptions of fitness. Frontiers in Genetics, $3: 1-12$.

Ariew, A., and Ernst, Z. 2009. What Fitness Can’t Be. Erkenntnis, 71:289-301. 
Ariew, A., and Lewontin, R.C. 2004. The confusions of fitness. The British Journal for the Philosophy of Science, 55:347-363.

Arnold, S.J. 1983. Morphology, performance and fitness. American Zoologist, 23:347-361.

Bouchard, F., and Rosenberg, A. 2004. Fitness, probability and the principles of natural selection. The British Journal for the Philosophy of Science, 55:693-712.

Brandon, R.N. 1978. Adaptation and evolutionary theory. Studies in the History and Philosophy of Science 9:181-206.

Camus, M.F., Clancy, D.J., and Dowling, D.K. 2012. Mitochondria, maternal inheritance, and male aging. Current Biology, 22:1717-1721.

Clutton-Brock, T. 1988. Reproductive Success: Studies of Individual Variation in Contrasting Breeding Systems. Chicago: University of Chicago Press.

Darwin, C. 1896. The descent of man and selection in relation to sex (Vol. 1). D. Appleton.

Darwin, C. 2004. On the Origin of Species, 1859. Routledge.

Dowling, D.K., and Adrian, R.E. 2019. Challenges and prospects for testing the mother's curse hypothesis. Integrative and Comparative Biology, 59:875-889.

Edwards, A. W. 1998. Natural selection and the sex ratio: Fisher's sources. The American Naturalist, 151:564-569.

Fisher, R. A. 1958. The genetical theory of natural selection. Oxford University Press. 
Foerster, K., Coulson, T., Sheldon, B.C., Pemberton, J.M., Clutton-Brock, T.H., and Kruuk, L.E.B. 2007. Sexually antagonistic genetic variation for fitness in red deer. Nature, 447:1107-1111.

Gemmell, N.J., Metcalf, V.J., and Allendorf, F.W. 2004. Mother's curse: the effect of mtDNA on individual fitness and population viability. Trends in Ecology and Evolution, 19:238-244.

Grafen, A. 2020. The Price equation and reproductive value. Philosophical Transactions of the Royal Society B, 375:20190356.

Hadfield, J.D., and Nakagawa, S. 2010. General quantitative genetic methods for comparative biology: phylogenies, taxonomies and multi-trait models for continuous and categorical characters. Journal of Evolutionary Biology, 23:494-508.

Harada, A.E., Healy, T.M., and Burton, R.S. 2019. Variation in thermal tolerance and its relationship to mitochondrial function across populations of Tigriopus californicus. Frontiers in Physiology, 10:213.

Hatefi, Y. 1985. The mitochondrial electron transport and oxidative phosphorylation system. Annual Review of Biochemistry, 54:1015-1069.

Heine, K.B., and Hood, W.R. 2020. Mitochondrial behaviour, morphology, and animal performance. Biological Reviews, 95:730-737.

Hill, G.E. 2020. Genetic hitchhiking, mitonuclear coadaptation, and the origins of mt DNA barcode gaps. Ecology and Evolution.

Hill, G.E. 2019. Mitonuclear Ecology. Oxford: Oxford University Press.

Hill, G.E. 2015. Mitonuclear ecology. Molecular Biology and Evolution, 32:1917-1927. 
Lane, N. 2006. Power, Sex, Suicide: Mitochondria and the Meaning of Life. Oxford: Oxford University Press.

Lime, T.G., Burton, R.S., and Willett, C.S. 2019. Genomic scans reveal multiple mito-nuclear incompatibilities in population crosses of the copepod Tigriopus californicus. Evolution, 73:609-620.

Lion, S. 2018. Class structure, demography, and selection: reproductive-value weighting in nonequilibrium, polymorphic populations. The American Naturalist, 191:620-637.

Luo, S., Valencia, C.A., Zhang, J., Lee, N.C., Slone, J., Gui, B., Wang, X., Li, Z., Dell, S., Brown, J., and Chen, S.M. 2018. Biparental inheritance of mitochondrial DNA in humans. Proceedings of the National Academy of Sciences, 115:13039-13044.

Matthen, M., and Ariew, A. 2002. Two ways of thinking about fitness and natural selection. Journal of Philosophy 99:55-83.

Michod, R.E. 2000. Darwinian dynamics: evolutionary transitions in fitness and individuality. Princeton University Press.

Mills, S.K., and Beatty, J.H. 1979. The propensity interpretation of fitness. Philosophy of Science, 46:263-286.

Millstein, R.L. 2006. Natural selection as a population-level causal process. British Journal for the Philosophy of Science 57:627-653.

Millstein, R.L. 2017. "Evolution.” The Stanford Encyclopedia of Philosophy. Edward N. Zalta (ed.), URL $=<$ https://plato.stanford.edu/entries/evolution/> 
Nagarajan-Radha, V., Aitkenhead, I., Clancy, D.J., Chown, S.L., and Dowling, D.K. 2020. Sexspecific effects of mitochondrial haplotype on metabolic rate in Drosophila melanogaster support predictions of the Mother's Curse hypothesis. Philosophical Transactions of the Royal Society B, 375:20190178.

Orr, H.A. 2009. Fitness and its role in evolutionary genetics. Nature Reviews Genetics, 10:531539.

Pence, C.H., and Ramsey, G. 2013. A new foundation for the propensity interpretation of fitness. The British Journal for the Philosophy of Science, 64:851-881.

Pence, C.H., and Ramsey, G. 2015. Is organismic fitness at the basis of evolutionary theory? Philosophy of Science, 82:1081-1091.

Ramsey, G. 2006. 'Block Fitness', Studies in History and Philosophy of Biological and Biomedical Sciences, 37:484-98.

Rosenberg, A., and Bouchard, F. 2015. "Fitness." The Stanford Encyclopedia of Philosophy. Edward N. Zalta (ed.), URL=<https://plato.stanford.edu/entries/fitness/>

Sober, E. 2013. Trait fitness is not a propensity, but fitness variation is. Studies in History and Philosophy of Science Part C: Studies in History and Philosophy of Biological and Biomedical Sciences, 44:336-41.

Strevens, M. 2016. The reference class problem in evolutionary biology: distinguishing selection from drift. Chance in Evolution. University of Chicago Press, 145-175.

Vaught, R.C., and Dowling, D.K. 2018. Maternal inheritance of mitochondria: Implications for male fertility? Reproduction, 155:R159-R168. 


\section{ACKNOWLEDGMENTS}

We wish to thank Marshall Abrams, Geoff Hill, Wendy Hood, Yoichi Ishida, Charles Pence, Matt Powers, Aaron Novick, and Matthew Wolak for helpful discussion and comments on an earlier draft of the paper. 